\title{
Editorial
}

\section{The limited value of measuring gene flow via errant pollen from GM plants}

\author{
Alan MCHUGHEN \\ Batchelor Hall 3110, University of California, Riverside, CA 92521-0124, USA \\ E-mail: alanmc@ucr.edu
}

\begin{abstract}
Since the advent over 30 years ago of recombinant DNA technologies giving rise to genetically engineered organisms (often called GMOs), gene escape from such transgenic organisms has been a consistent and legitimate concern. Initially, the fear of GM microbes escaping and wreaking ecological havoc on the biosphere generated both well funded scientific analyses of the actual threat, and fueled science fiction tracts of rampant monster microbes consuming every living thing on Earth. In recent years the focus-at least in the scientific community - has shifted to GM plants, particularly the incidence of escape of genes from GM crops.

The lion's share of the funding and research effort has gone to study gene escape via pollen dispersal. Each species has different pollen flow characteristics, from the vectors, to the distance, to the list of prospective recipient species and varieties. Some crops, like maize/corn and Brassica napus, produce large amounts of viable pollen and outcross notoriously; such species spawn plenty of research attention from both scientists and granting agencies. As a result, this journal and other literature is now teeming with data, models and evidence documenting the likelihood, mechanisms and incidence of pollenmediated gene flow from transgenic plants.

Most crop plants produce pollen, and pollen can be carried by wind, insects, animals or other vectors to distant locales where they may successfully pollinate a waiting recipient. The recipient could be another plant of the same species, but a different (GM or non-GM) cultivar, or it could be a compatible relative, with or without weedy characteristics. Depending on the nature of the gene and the recipient, the resulting hybrid may or may not cause concern. In the most benign situation, for example, a pollen grain from a GM or non-GM plant might blow or be carried across the road to pollinate another plant of the same cultivar growing in a neighbor's field.
\end{abstract}

Although this is clearly an example of gene escape and outcrossing, few scientists would get excited (let alone funded) to investigate and measure the incidence or implications of such events. Indeed, how would one even detect this eventuality, given the background of genetically identical proximal sources of pollen? How would one distinguish the pollination from an adjacent plant as opposed to the pollen grain of a genetically identical plant from across the road?

At the other end of the spectrum, we can envisage a situation in which gene escape is important, for example in plants engineered to produce pharmaceutical or potentially toxic industrial compounds. Similarly, genes conferring ecological fitness traits such as drought or salt tolerance would be problematic if the genes were to escape cultivation and wind up in plants out-competing other plants in unmanaged ecosystems. The main ecological hazard here is genetic proliferation and spread beyond intended borders. Increased ecological fitness is a real hazard and warrants considerable research. But not all GM genes confer fitness traits, and many fitness (or 'weedy') characteristics appear in non-GM plants.

The point of these investigations is to inform the scientific community, regulators and society at large of the relative hazards posed by GM crops. But the pollen flow studies, although necessary, are insufficient because (1) measures of pollen flow don't identify actual hazards (if any) posed as a consequence of the inevitable gene escape and (2) without some comparative data we don't whether pollen-based gene flow is a greater or lesser means of gene escape than other common routes (such as seed spillage or volunteerism). Unless and until we compare pollen-based gene flow with other means of gene escape, we cannot properly inform policymakers, and thus we are incomplete in our scientific assessments. 
A. McHughen

Any number of scientific teams seek and are awarded funds to investigate, using the latest mathematical models, molecular markers, wind tunnels and other tools to measure pollen flow, but how many research awards are given to scientific teams to measure gene escape caused by seed spillage at seeding or harvest time, inadvertent seed transport on machinery or mundane admixtures in farmyards and seed storage areas? Sure, quantifying seed spillage is tedious and mundane. Granting agencies are less likely to fund such projects. And few scientists are likely to get excited about conducting such projects. But if we want to know the actual incidence of gene escape, the information is necessary.

Say, for example, our latest research shows, within reasonable variation, that pollen escape and subsequent cross pollination from a given GM crop growing on a given farm in a given environment has a frequency value of $(x)$. Apart from situations where the gene in question is so noxious that prudence demands a ban from open environment cultivation in the first place (in which case pollen flow studies would be irrelevant anyway), how does the value $(x)$ provide any insight into either the biological consequence of gene flow or inform public policy for regulatory or educational purposes? Until we know how pollen based gene flow $(x)$ compares with gene escape frequencies from other mechanisms, we remain ignorant and continue to make poor policy decisions based on the singular data set. To illustrate, perhaps policymakers use gene flow $(x)$ value to adopt a mitigation policy to reduce the frequency to a level deemed acceptable, 0.01(x), the costs of implementation of that policy being $\$ \mathrm{M}$. With that mitigation policy implemented, farmers grow the said crop confident that the gene flow issue is resolved. But wait... What if the frequency of gene escape from non-pollen sources, e.g. seed spillage/volunteerism/theft of seed/ turns out to be $1000(x)$ ? The total actual gene escape is far greater than predicted from the pollen based studies. Not only is $\$ \mathrm{M}$ and implementation resources for a mitigation policy that may work perfectly as predicted in limiting pollen based gene flow wasted, we also suffer whatever the negative consequences might be from the actual gene escape. Although the pollen based gene flow information was scientifically accurate and led to a mitigation scheme that was also effective at restricting the pollen based gene flow, it failed to do what it was supposed to do in the first place, inform policy to protect against the hazards associated with gene escape.

Finally, the assumption that only GM plants pose hazards from gene escape must be challenged. All of the ecological damage wrought on this planet to date show nonGMOs to be responsible, so our focus on GM plants to the exclusion of non-GM is clearly wrong. Ecosystems are threatened by introductions of species from elsewhere, from natural invasions and successions and from expanding human intrusions (especially ordinary agriculture and urbanization). Focusing on one small component-GM agriculture-means we overlook the true threats to ecology and biological diversity.

Good policy demands scientifically complete and valid information. We need to consider both context and comparatives. Pollen flow studies are necessary, but by themselves are insufficient. Information needed, but currently lacking or underrepresented, includes whether gene escape incidence differs for transgenes compared to non-transgenes, and an assessment of gene escape frequencies from all routes, not only from errant pollen. Other crucial but missing information includes an assessment of the ecological consequences of the inevitable escape. These data must then be placed and evaluated in the context of all threats to ecosystems, including natural invasions and introductions of non-GM species. Only after assessing all of these data can good public and regulatory policy be forged. 